\title{
Prevalence of Feline Coronavirus Antibodies in Japanese Domestic Cats during the Past Decade
}

\author{
Satoshi TAHARAGUCHI ${ }^{1) *}$, Takehisa $\mathrm{SOMA}^{2)}$ and Motonobu HARA ${ }^{1)}$ \\ ${ }^{1)}$ Department of Microbiology II, School of Veterinary Medicine, Azabu University, 1-17-71 Fuchinobe, Chuo-ku, Sagamihara, \\ Kanagawa 252-5201, Japan \\ 2) Veterinary Diagnostic Laboratory, Marupi Lifetech Co., Ltd., 103 Fushiocho, Ikeda, Osaka 563-0011, Japan
}

(Received 26 December 2011/Accepted 18 May 2012/Published online in J-STAGE 1 June 2012)

ABSTRACT. From 2001 to 2010, 17,392 Japanese cats were examined for feline coronavirus (FCoV) antibodies. The seroprevalence of purebreds $(66.7 \%)$ was higher than that of random breds (31.2\%). Seroprevalence increased greatly in purebreds by three months of age, while it did not fluctuate greatly in random breds with aging, indicating that cattery environments can contribute to FCoV epidemics. Purebreds from northern regions of Japan were likely to be seropositive (76.6\% in Hokkaido, $80.0 \%$ in Tohoku), indicating cattery cats in cold climates might be more closely confined. Among purebreds, the American shorthair, Himalayan, Oriental, Persian, and Siamese showed low seroprevalence, while the American curl, Maine coon, Norwegian forest cat, ragdoll and Scottish fold showed high seroprevalence. There would also be breed-related differences in Japan similar to the previous studies in Australia.

KEY WORDS: domestic cat, feline coronavirus, Japan, seroprevalence.

doi: 10.1292/jvms.11-0577; J. Vet. Med. Sci. 74(10): 1355-1358, 2012

Feline coronavirus (FCoV) is a positive stranded RNA virus that has been classified as a Group 1 coronavirus along with canine coronavirus, transmissible gastroenteritis virus and human coronavirus 229E and infects domestic cats and wild felids. It is shed in feces by healthy cats, is transmitted by the fecal-oral route and causes mild to inapparent enteritis [7]. FCoV is of little clinical significance except when it undergoes a mutation that can lead to a highly fatal and currently untreatable systemic disease known as feline infectious peritonitis (FIP) [7]. Therefore, the incidence of FIP is directly related to the prevalence of FCoV infection in any given environment or geographic region. Studies on the prevalence of FCoV infection in Japan have been limited, and have not taken into consideration important variables such as breed, age, and environment $[7,15]$. To the best of our knowledge, no studies have been done in Japan regarding FCoV prevalence that have included a large-scale analysis of these variables. To this end, the present study analyzed FCoV seroprevalence among over 17,000 cats of various breeds, ages, genders and geographic origins across Japan.

Sera obtained from 17,392 one-month-to 20-year-old domestic cats brought to veterinary clinics for health checkups throughout Japan from January 2001 to August 2010 were examined for $\mathrm{FCoV}$ antibodies. Retest results from the same cats were excluded.

The antibody against FCoV was examined by a modification of the methods reported by Ishida et al. [9] and Kai et

\footnotetext{
*Correspondence to: Taharaguchi, S., Department of Microbiology II, School of Veterinary Medicine, Azabu University, 1-17-71 Fuchinobe, Chuo-ku, Sagamihara, Kanagawa 252-5201, Japan. e-mail: tsatoshi@azabu-u.ac.jp

(C)2012 The Japanese Society of Veterinary Science
}

al. [11]. In brief, $1.2 \times 10^{4}$ Crandell-Reese feline kidney cells/well were added to 96-well tissue culture plates and grown overnight at $37^{\circ} \mathrm{C}$. Each well was inoculated with 10 TCID $_{50}$ of an FIP virus (FIPV) that was isolated from ascites of a random bred cat with effusive FIP in Kanagawa, Japan, in 1996 [3]. This strain of FIPV was identified as FCoV type II using an anti-FCoV type II-specific cat polyclonal antibody (VMRD, Pullman, WA, U.S.A.) and a nested RT-PCR reported by Addie et al. [2]. Following $24 \mathrm{hr}$ incubation, cultured cells were fixed in the plate with methanol, washed with distilled water, dried and stored at $-20^{\circ} \mathrm{C}$ until used. Serum samples to be tested were diluted 1:200 with phosphate buffered saline solution with Tween 20 (PBST) (pH 7.0), and then $50 \mu l$ of the diluted sample was added to each well. Following one hr incubation at $37^{\circ} \mathrm{C}$, the plate was washed three times with PBST, and then $50 \mu l$ of peroxidase-conjugated goat anti-cat $\operatorname{IgG}(\mathrm{H}+\mathrm{L})$ (Jackson ImmunoResearch Laboratories, West Grove, PA, U.S.A.) diluted 1:1,500 with PBST containing $1 \%$ bovine serum albumin, was added to each well of the plate. After one hr incubation at $37^{\circ} \mathrm{C}$, the plate was washed three times with PBST, and then $100 \mu l$ of the substrate solution $\left[0.0025 \%\right.$ dianisidine, $0.01 \% \mathrm{H}_{2} \mathrm{O}_{2}$ and $10 \mathrm{mM}$ Tris- $\mathrm{HCl}$ (pH 7.4)] was added to each well. After 30 min incubation at $37^{\circ} \mathrm{C}$, the plate was washed with distilled water and then observed microscopically. Positive reactions judged as multiple brown-colored foci were observed.

Previous studies $[10,11]$ have shown that cats experimentally infected with FIPV or feline enteric coronavirus acquired an antibody titer of 1:200 or greater using a procedure similar to the present method. Therefore, an antibody titer of 1:200 or greater was considered to be seropositive in this study. However, this method cannot discriminate between maternal and active antibodies.

The significance of seroprevalence data was analyzed by 


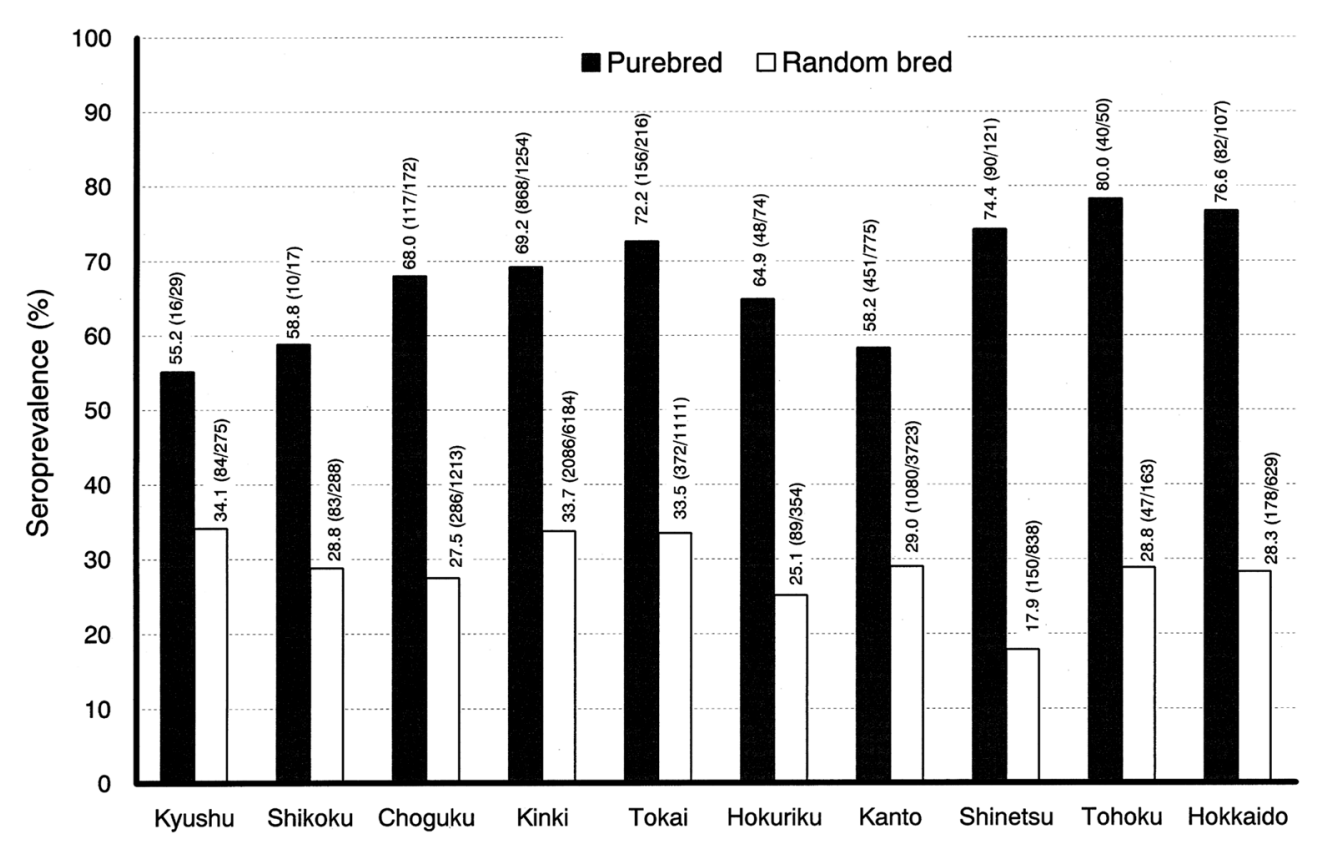

Fig. 1. FCoV seroprevalence of purebred and random bred cats, grouped by region of Japan. Black and white bars indicate the prevalence of purebred and random bred cats, respectively.

chi-square test using PC software (StatView, Adept Scientific, Letchworth, Herts, U.K.), and a $P$ value of $<0.05$ was considered statistically significant.

Of the 17,392 cats in this study, 6,433 (37.0\%) were seropositive for $\mathrm{FCoV}$. The seroprevalences for purebred and random bred cats were $66.7 \%(1,878$ of 2,815$)$ and $31.2 \%$ $(4,555$ of 14,577), respectively, and were significantly different $\left(P<0.0001, \chi^{2}=1273.3\right)$. No significant differences in seroprevalence were observed between males (purebred $66.3 \%, 1,015$ of 1,530 ; random bred $31.2 \%, 2,317$ of 7,430 ) and females (purebred $69.4 \%, 892$ of 1,285 ; random bred $30.9 \%, 2,209$ of 7,147). Furthermore, there was also no significant difference in seroprevalence on a year to year basis (data not shown).

As shown in Fig. 1, seroprevalence among purebred cats in the northern regions of Japan (Hokkaido and Tohoku) was significantly higher $\left(76.6 \%, P=0.0321, \chi^{2}=4.6\right.$, and $80.0 \%$ $P=0.0477, \chi^{2}=3.9$, respectively) than the average for purebred cats across Japan (66.7\%). Among random bred cats, seroprevalence was significantly higher in the Kinki region $\left(P=0.0004, \chi^{2}=12.3\right)$ than the average among random bred cats across Japan (30.6\%), while those in Chugoku, Hokuri$\mathrm{ku}$, Kanto and Shinetsu regions were significantly lower than the average $\left(P<0.0001, \chi^{2}=31.0 ; P=0.0142, \chi^{2}=6.0\right.$; $P=0.0083, \chi^{2}=7.0$; and $P<0.0001, \chi^{2}=66.6$, respectively).

Seroprevalence among purebred cats progressively increased to $80 \%$ at 3 months of age and remained at this level until 2 years of age (Figs. 2 and 3). It declined thereafter and reached nearly the same level as for random breds at around 11 years of age (Fig. 3). By contrast, seroprevalence among random bred cats remained at around $30 \%$, a pattern quite different from that of the purebred cats.

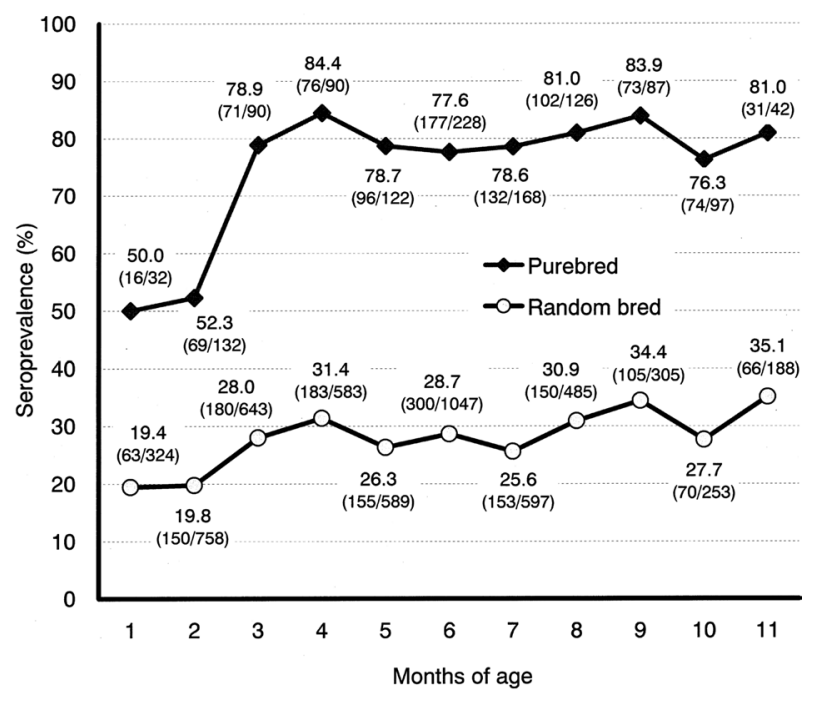

Fig. 2. FCoV seroprevalence of purebred and random bred cats under one year of age, grouped by age in months. Diamonds and circles indicate the prevalence of purebred and random bred cats, respectively.

Seroprevalence was also compared among various breeds of purebred cats. As shown in Table 1, the American curl, Maine coon, Norwegian forest cat, ragdoll and Scottish fold breeds showed high seroprevalence, whereas the American shorthair, Himalayan, Oriental, Persian and Siamese breeds showed low seroprevalence.

The present results obtained from an FCoV antibody test 


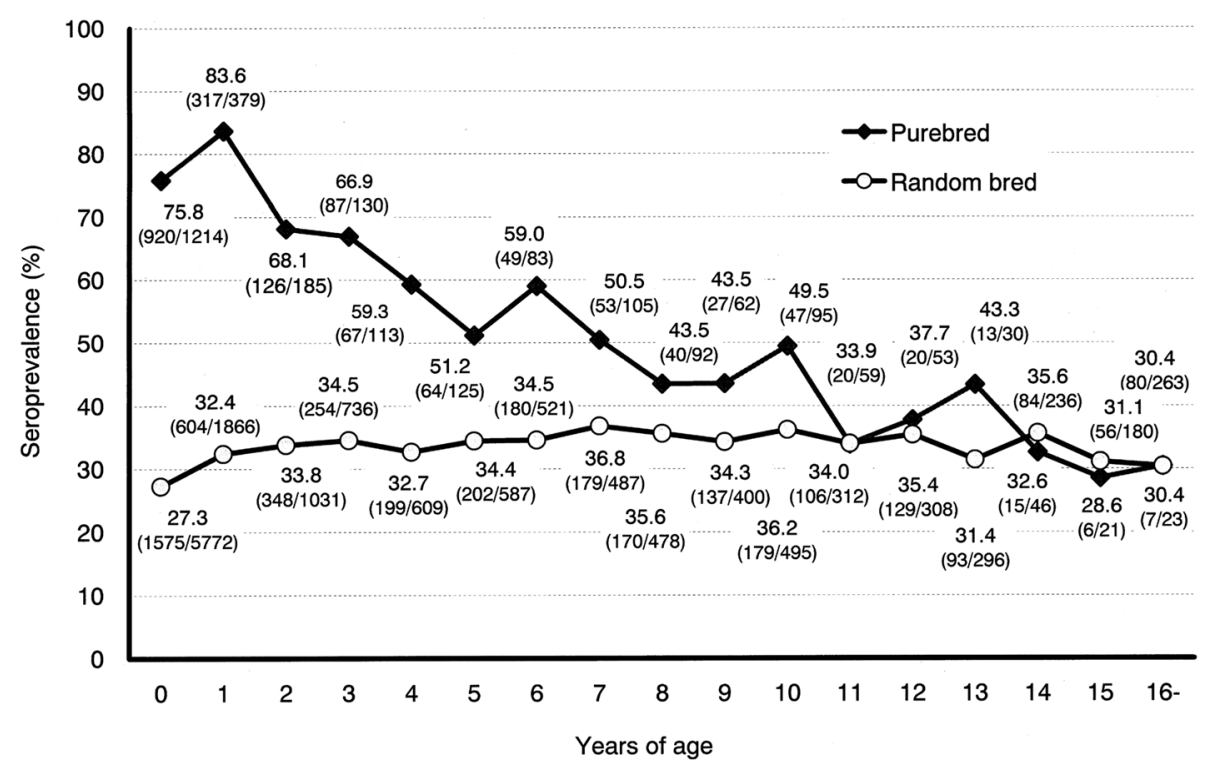

Fig. 3. FCoV seroprevalence of purebred and random bred cats, grouped by age. Diamonds and circles indicate the prevalence of purebred and random bred cats, respectively.

Table 1. FCoV seroprevalence among various breeds in purebred cats

\begin{tabular}{|c|c|c|}
\hline Breed & Seroprevalence & $\begin{array}{c}P \text { value } \\
\text { (vs average of purebreds) }\end{array}$ \\
\hline Abyssinian & $68.10 \%$ (113 of 166$)$ & $>0.05$ \\
\hline American curl & $83.30 \% \quad(30$ of 36$)$ & $0.0352\left(\chi^{2}=4.4\right)$ \\
\hline American shorthair & $59.70 \% \quad(420$ of 703$)$ & $0.0005\left(\chi^{2}=12.1\right)$ \\
\hline Bengal & $70.00 \% \quad(21$ of 30$)$ & $>0.05$ \\
\hline Himalayan & $57.50 \% \quad(73$ of 127$)$ & $0.00313\left(\chi^{2}=4.6\right)$ \\
\hline Maine coon & $75.00 \%(135$ of 180$)$ & $0.0217\left(\chi^{2}=5.3\right)$ \\
\hline Norwegian forest cat & $91.60 \% \quad(88$ of 96$)$ & $<0.0001 \quad\left(\chi^{2}=26.4\right)$ \\
\hline Oriental & $22.40 \%(11$ of 49$)$ & $<0.0001 \quad\left(\chi^{2}=42.0\right)$ \\
\hline Persian & $61.40 \%(258$ of 420$)$ & $0.0329\left(\chi^{2}=4.6\right)$ \\
\hline Ragdoll & $88.10 \% \quad(59$ of 67$)$ & $0.0002\left(\chi^{2}=13.5\right)$ \\
\hline Russian blue & $68.40 \% \quad(199$ of 291$)$ & $>0.05$ \\
\hline Scottish fold & $79.40 \%$ (313 of 394$)$ & $<0.0001 \quad\left(\chi^{2}=27.7\right)$ \\
\hline Siamese & $39.60 \% \quad(21$ of 53$)$ & $<0.0001 \quad\left(\chi^{2}=15.9\right)$ \\
\hline Somali & $66.30 \% \quad(59$ of 89$)$ & $>0.05$ \\
\hline Others & $68.40 \%$ (78 of 114$)$ & \\
\hline Purebreds (avarege) & $66.70 \% \quad(1,878$ of 2,815$)$ & \\
\hline
\end{tabular}

The breeds that consisted of fewer than 30 cats were classified together into others. Each breed was compared with the average for the purebred cats $(66.7 \%)$ by chi-square test.

conducted with over 17,000 domestic cats from across Japan show in detail the prevalence of FCoV infection in Japan, which has not previously been fully understood.

No difference in seroprevalence was observed between males and females, similar to previous studies $[4,8]$. This finding supports the ease by which $\mathrm{FCoV}$ is transmitted among cats $[7,13]$.

It has been reported that maternal antibodies can be detected by 10 weeks of age [6], yet the present method cannot discriminate between maternal and active antibodies.
However, the influence of maternal antibodies on the present analysis would be negligible, since the seroprevalence at 1-2 months of age, which is when maternal antibodies may be detected $[1,13]$, was extremely low compared with that at 3-11 months of age. A markedly higher seroprevalence was observed in purebred cats compared with random bred cats. Studies in other parts of the world have also shown that the $\mathrm{FCoV}$ infection rate was higher in purebred cats than in random bred cats [8]. This discrepancy would be attributed to the following things. It has been reported that most kittens 
in catteries become infected at around 6 to 10 weeks of age $[1,13]$. A similar trend was seen among purebred cats in the present study, with seroprevalence progressively rising during this same age period. Juvenile purebred cats are reared mainly in multi-cat environments, such as breeding catteries and pet shops. These types of environments contribute to $\mathrm{FCoV}$ exposure in Japan in the same manner as in other countries $[7,15]$.

The present finding that the seroprevalence of purebred cats declined from 2 to 11 years of age shows that only a small number of adult purebred cats would be reinfected after elimination of the carrier status. Furthermore, the seroprevalence of random bred cats, which are unlikely to be kept in catteries, was approximately constant at around 30\%. The above findings indicate that household cats would be at extremely low risk of $\mathrm{FCoV}$ infection, as previously noted [15].

It was noteworthy that $\mathrm{FCoV}$ exposure among purebred cats was greater in northern climates, where cattery cats are more closely confined due to colder weather. Unlike purebred cats, no uneven distribution of low or high seroprevalence was observed among random bred cats, although significantly higher prevalence was observed in the Kinki region and significantly lower prevalence was observed in Chugoku, Hokuriku, Kanto and Shinetsu regions.

Similar to the previous studies in Australia [4, 5], the Siamese and Persian breeds showed significantly low seroprevalence in the present study. These are "classic breeds" that have been in existence for more than a century after establishment as pedigree breeds. On the other hand, most of the breeds that showed high seroprevalences in the present study, and cats that demonstrated a high frequency of FCoV antibody production and FIP manifestation in previous studies were found to be purebreds recently $[4,5,12,14]$. Unlike the Siamese and Persian, for maintaining pure lines of these breeds frequent movements of breeding cats between breeding catteries and inbreeding are more likely, which might affect the $\mathrm{FCoV}$ endemic and resistance to FIP.

From above findings, nationwide virological studies in multi-cat environments such as rescue catteries, breeding catteries and pet shops are necessary to find some methods for prevention of FCoV infections in Japan. In addition, the source of breeding cats should also be verified in the future.

\section{REFERENCES}

1. Addie, D. D. and Jarrett, J. O. 1992. Feline coronavirus antibodies in cats. Vet. Rec. 131: 202-203. [Medline] [CrossRef]

2. Addie, D. D., Schaap, I. A., Nicolson, L. and Jarrett, O. 2003. Persistence and transmission of natural type I feline coronavirus infection. J. Gen. Virol. 84: 2735-2744. [Medline] [CrossRef]

3. Arifuku, K. and Hara, M. 1997. A cases of feline infectious peritonitis manifested post otitis crouposa. J Environ. Dis. 4: 11-14.

4. Bell, E. T., Malik, R. and Norris, J. M. 2006. The relationship between the feline coronavirus antibody titre and the age, breed, gender and health status of Australian cats. Aust. Vet. J. 84: 2-7. [Medline] [CrossRef]

5. Bell, E. T., Toribio, J. A., White, J. D., Malik, R. and Norris, J. M. 2006. Seroprevalence study of feline coronavirus in owned and feral cats in Sydney, Australia. Aust. Vet. J. 84: 74-81. [Medline] [CrossRef]

6. Gunn-Moore, D. and Addie, D. 2001. The peritoneal cavity. pp.151-166. In: Manual of Canine and Feline Infectious Diseases (Ramsey, I. K. and Tennant, B. J. eds.), British Small Animal Veterinary Association, Gloucester.

7. Hartmann, K. 2005. Feline infectious peritonitis. Vet. Clin. North Am. Small Anim. Pract. 35: 39-79. [Medline] [CrossRef]

8. Holst, B. S., Englund, L., Palacios, S., Renstrom, L. and Berndtsson, L. T. 2006. Prevalence of antibodies against feline coronavirus and Chlamydophila felis in Swedish cats. J. Feline Med. Surg. 8: 207-211. [Medline] [CrossRef]

9. Ishida, T., Washizu, T., Fukuoka, J., Toriyabe, K., Uchino, T. and Motoyoshi, S. 1987. Feline infectious peritonitis virus antibody test using enzyme-linked immunosorbent assay. Jpn. J. Vet. Sci. 49: 145-149. [Medline]

10. Kai, K., Akagi, Y., Soma, T., Nomura, K. and Kanoe, M. 1995. Biphasic immune responses of cats under controlled infection with a feline enteric coronavirus-79-1683 strain. J. Vet. Med. Sci. 57: 781-783. [Medline] [CrossRef]

11. Kai, K., Yukimune, M., Murata, T., Uzuka, Y., Kanoe, M. and Matsumoto, H. 1992. Humoral immune responses of cats to feline infectious peritonitis virus infection. J. Vet. Med. Sci. 54: 501-507. [Medline] [CrossRef]

12. Norris, J. M., Bosward, K. L., White, J. D., Baral, R. M., Catt, M. J. and Malik, R. 2005. Clinicopathological findings associated with feline infectious peritonitis in Sydney, Australia: 42 cases (1990-2002). Aust. Vet. J. 83: 666-673. [Medline] [CrossRef]

13. Pedersen, N. C. 1995. An overview of feline enteric coronavirus and infectious peritonitis virus infections. Feline Pract. 23: 7-20.

14. Pesteanu-Somogyi, L. D., Radzai, C. and Pressler, B. M. 2006. Prevalence of feline infectious peritonitis in specific cat breeds. J. Feline Med. Surg. 8: 1-5. [Medline] [CrossRef]

15. Wolf, A. M. 1997. Feline infectious peritonitis, part 2. Feline Pract. 25: 24-28. 